\title{
Molecular dynamics investigation with the time resolved optical Kerr effect on the $\mathrm{CS}_{2}-\mathrm{C}_{6} \mathrm{H}_{6}$ mixtures
}

Cite as: J. Chem. Phys. 125, 184503 (2006); https://doi.org/10.1063/1.2371110

Submitted: 31 August 2006 . Accepted: 29 September 2006 . Published Online: 09 November 2006

Ismael A. Heisler, Ricardo R. B. Correia, and Silvio L. S. Cunha

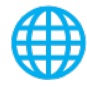

\section{ARTICLES YOU MAY BE INTERESTED IN}

Time-resolved optical Kerr-effect investigation on $C S_{2}$ /polystyrene mixtures

The Journal of Chemical Physics 123, 054509 (2005); https://doi.org/10.1063/1.1994850

Isotropic and anisotropic Raman scattering from molecular liquids measured by spatially masked optical Kerr effect spectroscopy

The Journal of Chemical Physics 117, 1139 (2002); https://doi.org/10.1063/1.1485070

Dielectric relaxation of electrolyte solutions using terahertz transmission spectroscopy

The Journal of Chemical Physics 116, 8469 (2002); https://doi.org/10.1063/1.1468888

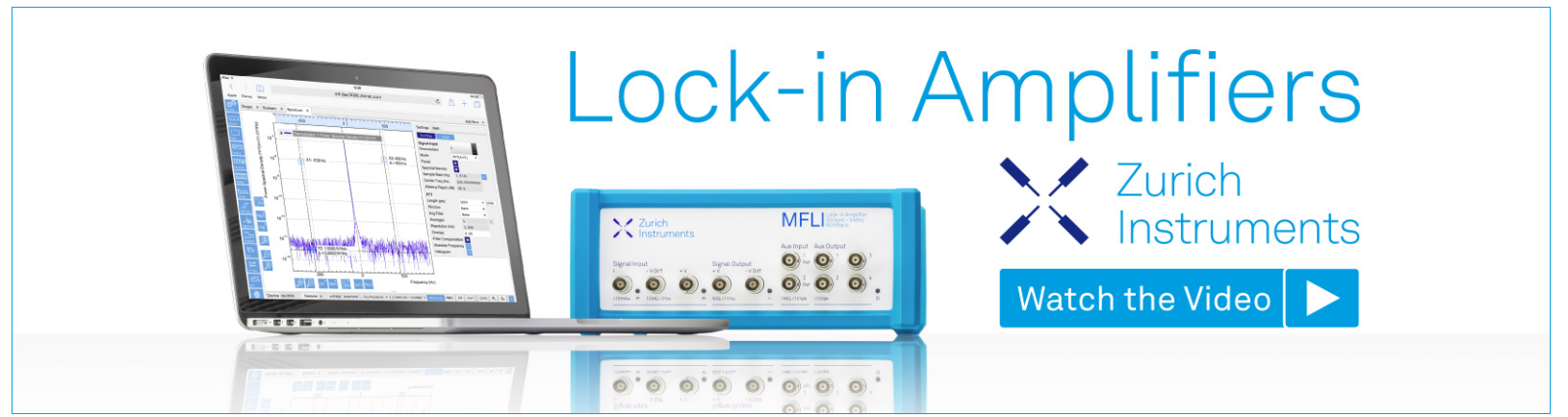




\title{
Molecular dynamics investigation with the time resolved optical Kerr effect on the $\mathrm{CS}_{2}-\mathrm{C}_{6} \mathrm{H}_{6}$ mixtures
}

\author{
Ismael A. Heisler, ${ }^{\text {a) }}$ Ricardo R. B. Correia, and Silvio L. S. Cunha \\ Instituto de Física, Universidade Federal do Rio Grande do Sul-UFRGS, Avenida Bento Gonçalves, 9500, \\ Caixa Postal 15051, CEP 91501-970, Porto Alegre, RS, Brazil
}

(Received 31 August 2006; accepted 29 September 2006; published online 9 November 2006)

\begin{abstract}
An investigation of the molecular dynamics in pure liquids and in mixtures through the technique of time resolved optical Kerr effect is performed. The samples studied were the mixtures of carbon disulfide $\left(\mathrm{CS}_{2}\right)$ with benzene $\left(\mathrm{C}_{6} \mathrm{H}_{6}\right)$. The molecular dynamics of the pure liquids is briefly discussed while the main results are obtained for the mixtures. A slow dynamics component is observed for the optical heterodyne detected optical Kerr effect transient decaying exponentially with time constants on picosecond time scale. The fast subpicosend time relaxations are analyzed in terms of the nondiffusive component of the spectral response that is associated with the molecular dynamics. The modifications of the spectrum are quantified, and the explanation of the observed changes is given in terms of the structural interaction configurations that produced changes in the intermolecular potential within which the molecules execute librational motions. () 2006 American Institute of Physics. [DOI: 10.1063/1.2371110]
\end{abstract}

\section{INTRODUCTION}

The low frequency domain of the spectral density associated to the liquid state, obtained with light scattering techniques and its time domain analogues, as the time resolved optical Kerr effect, is a peculiar region where the discrimination among different relaxation processes is not so far clearly defined. The processes originating the spectra in the low frequency region are tentatively separated into molecular reorientations, collision induced polarizability anisotropy, dipole-induced dipole interactions, and intermolecular coherent motions. ${ }^{1}$ The difficulties faced in interpreting intermolecular spectra derive from the fact that not all the abovementioned processes can be identified in an unambiguous manner by acting on classical experimental variables such as temperature or pressure, for example. In the recent past, many papers were published concerning investigations of the dynamics of pure simple liquids, such as carbon disulfide and benzene. ${ }^{2-5}$ The aim of those investigations was to disclose the dynamical behavior underlying the molecular motions in liquids. Neat liquid systems were extensively studied under the limitations of optical Kerr effect (OKE) and, therefore the picture of a molecular probe has added new perspectives into the intermolecular dynamics. New investigations were focused on the dynamical behavior in liquid mixtures, providing a more complete picture of the interactions occurring in solutions and of the solvent effect on intra- and intermolecular modes. As discussed by Steffen et al. ${ }^{6}$ an aspect that has to be accounted in a description of liquid state dynamics is the effect of damping processes on the optical response. The dephasing, due to random interactions with the bath, contributes significantly in altering the spectral width. The mathematical model that describes the spectral response function obtained through an OKE measure is influenced by

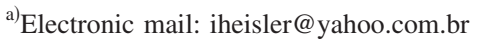

the dephasing processes. One motivation of current research in laboratories around the world is to elucidate the nature and mechanisms of the dephasing processes, because they underlie the complex microscopic dynamics in the liquid state. A common definition used throughout the literature is the one that classifies dephasing processes as homogeneous or inhomogeneous. The latter is due to a static distribution of local environments of molecules, which do not change on the time scale of the experiment, while homogeneous broadening is caused by fast fluctuations, which change the local environment on a time scale much faster than the inverse width of the frequency response. Since nuclear motions in liquids occur over a wide range of time scales, the separation into "fast" and "slow" fluctuations is questionable, and hence it is not clear whether it is fruitful to discuss the optical response only in extreme terms of homogeneous and inhomogeneous broadening.

Taking to account these facts, it would be of great interest to separate the inhomogeneous, structural and homogeneous, dynamic contributions to the spectral line shape of the intermolecular dynamics in the liquid state. Unfortunately this turns out not to be possible in optical heterodyne detected optical Kerr effect (OHD-OKE) measurements, which have only a single time dimension (the pump \pm probe delay) as was discussed by Tanimuran and Mukamel. ${ }^{7}$ It has been shown that such a separation is, in principle, possible in higher-order polarization dependent experiments such as, for example, in temporally two-dimensional experiments. ${ }^{7,8}$ The best known of these is the three-pulse photon echo, ${ }^{9,10}$ but the difficulties of performing this experiment at the far-IR frequencies required are considerable. Alternative six-wave mixing experiments have been proposed. ${ }^{6,7,11}$ Resonant sixwave mixing experiments have been demonstrated for studies of solute dynamics, ${ }^{12,13}$ but the nonresonant multidimensional experiments required to unravel the ground-state 
dynamics of liquids have proved challenging, in particular, because the six-wave signal is polluted by contributions from cascaded lower-order signals. ${ }^{12,13}$ In virtue of this, the OHDOKE continues to be a good alternative to obtain information about the intermolecular interactions. A good option is to analyze what happens in mixtures of molecules that have relatively strong interactions, observing the changes introduced to the spectral line shape in comparison to the pure liquids.

In a recent paper, we described the results obtained with the OHD-OKE technique for carbon disulfide-polystyrene $\left(\mathrm{CS}_{2}-\mathrm{PS}\right)$ mixtures. ${ }^{14}$ Through variations in the concentration of the mixture, it was possible to alter the effective intermolecular potential in a nearly continuous fashion and evaluate the main modifications introduced to the intermolecular dynamics. Our results showed that as the PS concentration was increased the principal librational frequency shifted to a higher frequency, consistent with a strengthening of the intermolecular forces between the phenyl rings of the PS molecules and the $\mathrm{CS}_{2}$ molecules. This association was corroborated by the fact that as $\mathrm{CS}_{2}$ molecules were replaced by the more strongly interacting phenyl rings, and the net effect was to modify the curvature of the intermolecular potential.

In this work we present experimental results obtained with the OHD-OKE technique performed on a series of carbon disulfide-benzene $\left(\mathrm{CS}_{2}-\mathrm{C}_{6} \mathrm{H}_{6}\right)$ mixtures. The objective is to gather information about the physical processes that underlie intermolecular dynamics in liquid mixtures and trace a broader picture concerning the dynamical processes subjacent to the complex $\mathrm{CS}_{2}-\mathrm{PS}$ mixtures. The $\mathrm{CS}_{2}-\mathrm{C}_{6} \mathrm{H}_{6}$ mixtures are intended to provide information about interactions between the $\mathrm{CS}_{2}$ molecules with the phenyl rings, which are the principal interacting structures present in the PS molecules. Both $\mathrm{CS}_{2}$ and $\mathrm{C}_{6} \mathrm{H}_{6}$ are molecules with strong polarizability anisotropy and these pure components have been studied in detail before. ${ }^{2,5,15}$ There are only a few experimental studies ${ }^{16,17}$ and molecular dynamics simulations ${ }^{18,19}$ which deal with the interpretation of the induced polarizability anisotropies in binary liquid mixtures. These studies are mostly associated with investigations of a strongly anisotropic component (i.e., $\mathrm{CS}_{2}$ or $\mathrm{C}_{6} \mathrm{H}_{6}$ ) diluted in an isotropic solvent (such as $\mathrm{CCl}_{4}$ and alkanes). ${ }^{2,4,20-22}$ In this situation, the analysis of the spectra is simplified by the fact that only reorientational fluctuations of the anisotropic component contribute to the spectra of the mixtures. On the other hand, the OHD-OKE transient signal of $\mathrm{CS}_{2}-\mathrm{C}_{6} \mathrm{H}_{6}$ mixtures appear as a sum of the contributions of the "total permanent" as well as of the "interaction induced" polarizability anisotropies arising from fluctuations of each liquid, complicating the results analysis.

\section{EXPERIMENT}

The ultrafast OHD-OKE measurements were carried out using linearly polarized laser pulses with duration of approximately $100 \mathrm{fs}$ and with a center wavelength of $780 \mathrm{~nm}$ (the pulse was characterized by the modified grating eliminated no-nonsense observation of ultrafast incident laser

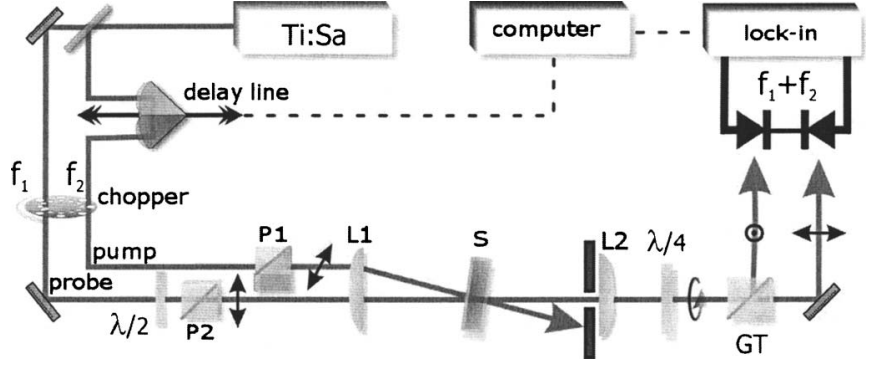

FIG. 1. Experimental setup. P1-2: polarizers, L1-2: lenses, S: sample, and GT: Glan-Thompson polarizer. $\lambda / 2$ and $\lambda / 4$ are zero-order half-wave retardation and quarter-wave retardation plates, respectively.

light E-fields (GRENOUILLE) method ${ }^{23}$ and the temporal envelope is well described by a square root hyperbolic secant). The pulses were generated in a commercial Kerr-lens mode-locked Ti:Sapphire laser (Mira 900 Coherent) pumped by a $5 \mathrm{~W}(\mathrm{cw})$ intracavity-doubled diode-pumped $\mathrm{Nd}: \mathrm{YVO}_{4}$ laser (Coherent Verdi). The $250 \mathrm{~mW}$ femtosecond laser beam was split into pump (95\%) and probe (5\%) beams, and the pump beam was optically delayed using a stepper motor resulting in a $5.6 \mathrm{fs} /$ step delay resolution (Fig. 1). Once the polarization of pump beam was determined, a polarizer was placed in the probe beam to achieve a $45^{\circ}$ angle relative to the pump. A $20 \mathrm{~cm}$ focal-length lens was used to focus the two beams into the sample. To enhance the sensitivity of the signal detection we used a phase-sensitive lock-in amplifier with a dual-slot chopper modulating the pump beam at $1.38 \mathrm{KHz}$ and the probe beam at $1.02 \mathrm{KHz}$. The signal was detected at the sum frequency of $2.4 \mathrm{KHz}$. At the sample position, the pulse temporal width was measured with a twophoton absorption photodiode. The ultrafast birefringence signal was obtained with a shot-noise limited, balanceddetection scheme, as described elsewhere. ${ }^{24}$ In this setup, the probe beam was circularly polarized with a quarter-wave retardation plate after the sample. Parallel and perpendicular components were separated with a Glan-Thompson polarizer and sent to a pair of photodiodes connected to the input channels of the lock-in amplifier. The signal obtained by electronically subtracting the horizontal from the vertical component canceled out both homodyne and background components. In turn, the pure heterodyne signal was recorded in a single shot. In addition, balanced detection reduces the effects of random fluctuations of the laser power and achieves a significant improvement in the signal to noise ratio.

Carbon disulfide and benzene of spectroscopic grade were obtained from Merck and used without further purification. The samples were prepared in glass cuvettes following a filtration of the liquid mixtures through a $22 \mu \mathrm{m}$ Millipore filter. All measurements were carried out at a temperature of $293 \mathrm{~K}$.

\section{RESULTS AND DISCUSSION}

The time resolved OKE experiment measures the time evolution of the optically induced third-order polarization anisotropy and, to a certain extent, has the capability to separate the different physical processes that lead the system to 

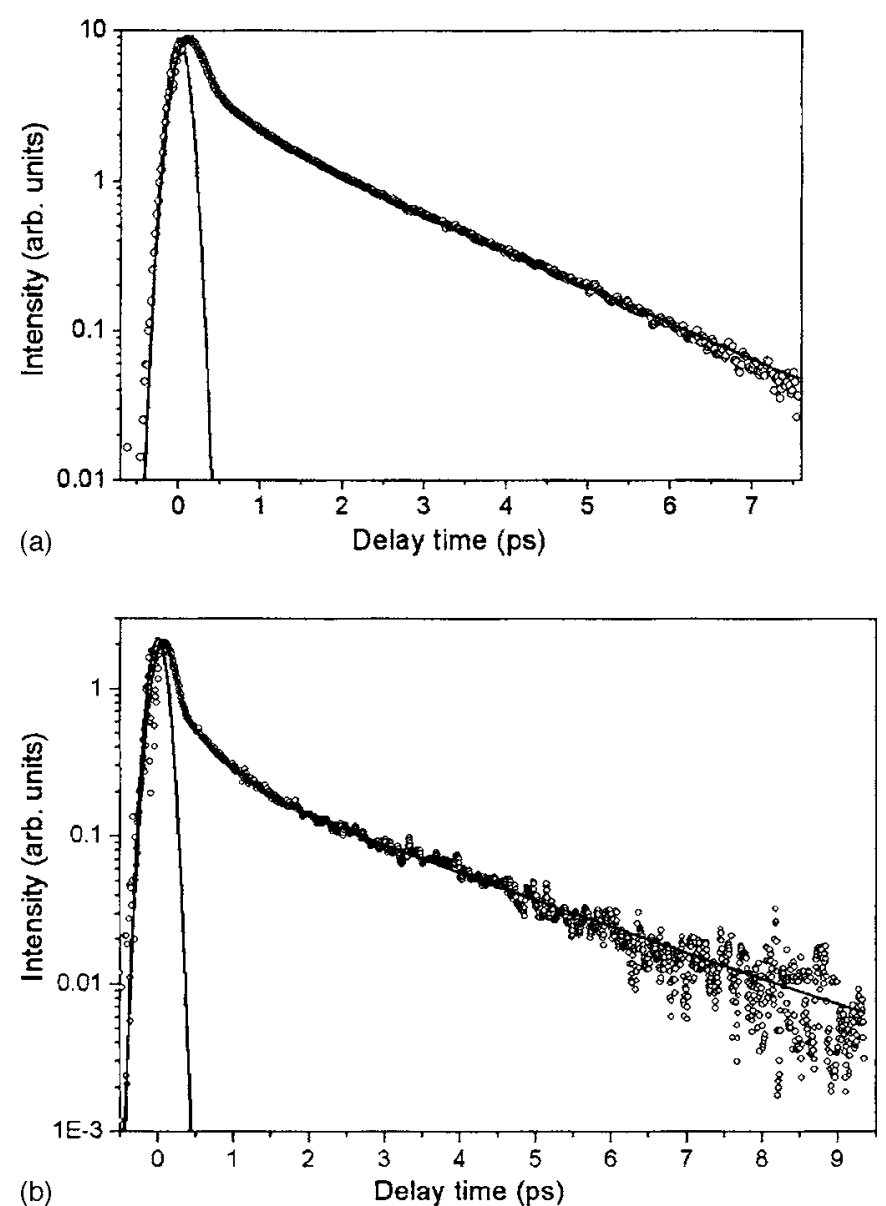

FIG. 2. OHD-OKE signal for neat (a) $\mathrm{CS}_{2}$ and (b) $\mathrm{C}_{6} \mathrm{H}_{6}$. Transients measured at $293 \mathrm{~K}$ (circles) together with the fitted molecular dynamics response.

relax on different time scales. At long times (typically a few picoseconds in liquids) the motion is generally described by orientational diffusion. For simple molecules such as carbon disulfide and benzene the experimental results can be interpreted on the basis of the Debye-Stokes-Einstein hydrodynamic theory. ${ }^{25}$ Figures 2 (a) and 2(b) show the OHD-OKE signal on a semilog scale together with the fitted nonlinear response functions for the carbon disulfide and the benzene molecules, respectively. The experimental transient signals were fitted to time response functions using an approach based on an evolutionary algorithm described in detail in Ref. 14. The long time diffusive component was fitted to an exponential function giving a time of $1.75 \pm 0.06 \mathrm{ps}$ for pure carbon disulfide and $2.24 \pm 0.11$ ps for pure benzene. This relaxation times confirm the well known literature results for these liquids. ${ }^{21,26,27}$ At shorter times (typically 100-1000 fs) the dynamics consist of complex, nondiffusive intermolecular motions (degrees of freedom) associated with the potential formed by nearby molecules through intermolecular forces. There are various contributions ${ }^{28}$ to the nondiffusive response (albeit yet neither all known nor well separated): interaction induced anisotropy (I-I) (including binary collisions) that arises from distortions of the molecular polarizabilities induced by the intermolecular interactions, intermolecular vibrational which include orientational (librational) and translational degrees of freedom, molecular frame distor- tions associated with molecular collisions, and others. It is a widespread common sense that the libration-type motion is the most significant contribution to the subpicosecond dynamics, and we will refer to the nondiffusive contribution to the data collectively as the "librational response" for the remainder of the paper. To describe the nondiffusive part of the nuclear response, we follow the Fourier transform deconvolution procedure developed by McMorrow ${ }^{29}$ and McMorrow and Lotshaw. ${ }^{30}$ Briefly, taking the Fourier transform of the OHD-OKE signal we have

$$
\mathfrak{I}\{I(t)\}=\mathfrak{I}\left\{A_{c}(t)\right\} \Im\{R(t)\},
$$

where we used the property of the Fourier transform for the convolution. In this expression, $I(t)$ is the measured OHDOKE signal, $A_{c}(t)$ is the independently measured intensity autocorrelation, and $R(t)$ is the nonlinear response function. The deconvolution is accomplished by computing the complex quotient,

$$
\frac{\mathfrak{I}\{I(t)\}}{\mathfrak{I}\left\{A_{c}(t)\right\}}=\mathfrak{I}\{R(t)\} \equiv D(\omega) .
$$

Since the very fast electronic contribution is simply described by a real constant that multiplies a Dirac delta function, this will not contribute to the imaginary part of the spectral distribution. ${ }^{30}$ So, as modeled, the information about all possible nuclear motions is contained in the imaginary part of the spectral distribution $\operatorname{Im}\{D(\omega)\}$. To better access the nondiffusive nuclear part, we subtracted the diffusive component prior to the deconvolution procedure. Figures 3(a) and 3(b) show the nuclear spectrum obtained after the deconvolution procedure of the measured experimental results for carbon disulfide and benzene, respectively. This spectra are "smooth," that is, do not show peaks or protuberances that can indicate a particular resonance or physical process, so the analyses of the data can become complex. Two parameters were chosen to do the characterization of such smooth asymmetrical spectra: the full width at half maximum $\Delta$ and the principal frequency or first moment of the spectral distribution $\langle\omega\rangle$. The values obtained for this parameters for the $\mathrm{CS}_{2}$ molecule were $\langle\omega\rangle=43.36 \mathrm{~cm}^{-1}$ and $\Delta=50.57 \mathrm{~cm}^{-1}$. For the benzene molecule the spectrum parameters obtained were $\langle\omega\rangle=65.08 \mathrm{~cm}^{-1}$ and $\Delta$ $=99.11 \mathrm{~cm}^{-1}$. In a liquid, where the mean free path before collisions is small, the molecules do not execute complete rotations and the frequencies of the librational motion are primordially determined by the moment of inertia. ${ }^{26}$ The moment of inertia for rotations around the axes that produces changes to the anisotropic ellipsoid is higher for the $\mathrm{CS}_{2}$ molecules in comparison to the $\mathrm{C}_{6} \mathrm{H}_{6}$ molecules. ${ }^{26}$ According to this model, $\mathrm{CS}_{2}$ molecules have a lower librational frequency and this originates a narrower spectral band, as measured by the $\Delta$ parameter.

Having presented the results for the pure liquids, now we turn to the analysis of the mixtures. There are at least two main reasons, which motivate the study of liquid mixtures. The first is to better characterize the dynamics of the pure liquid by, for example, using a dilution to minimize interaction induced contributions to the line shape. In this case a "noninteracting" solvent is often chosen as the second com- 

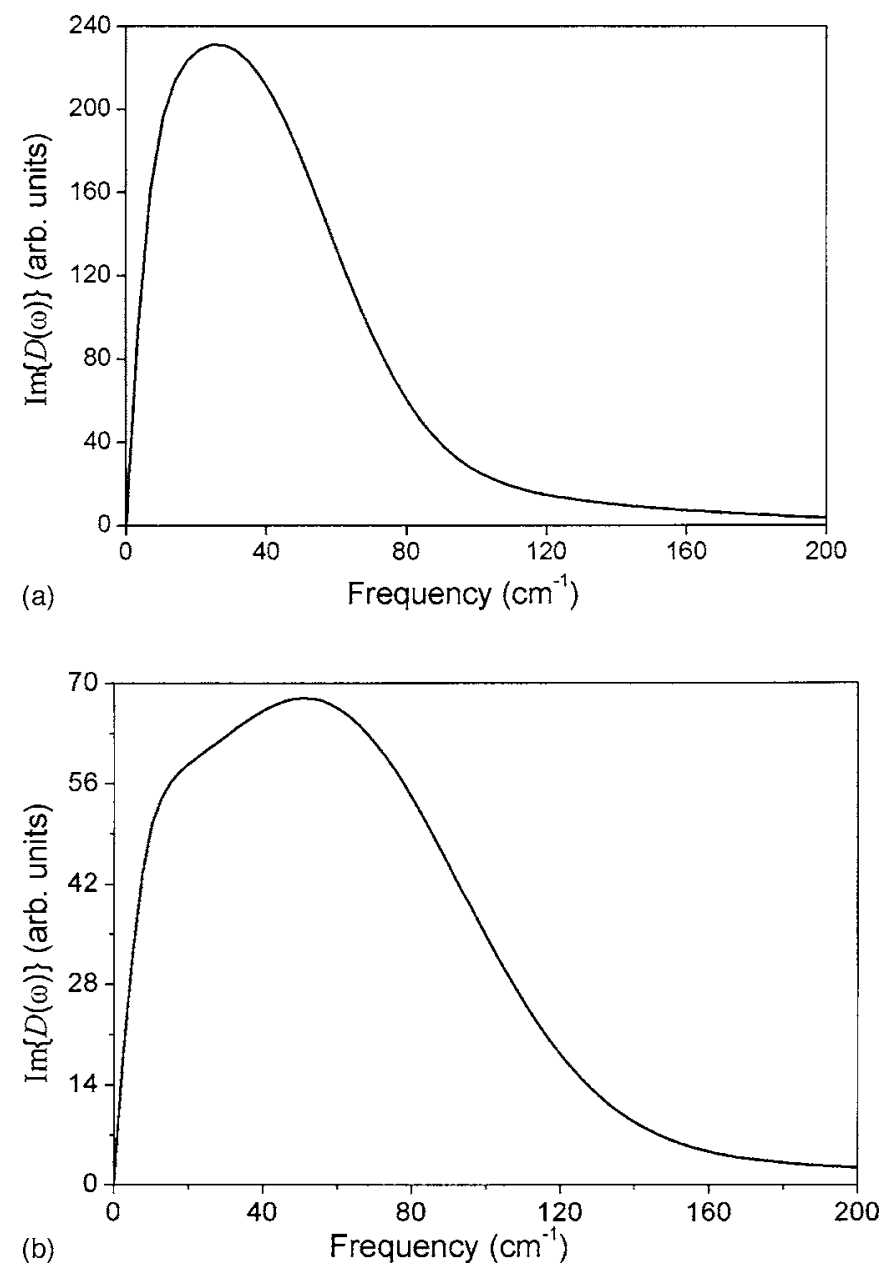

FIG. 3. Low frequency spectral distributions with the diffusive component subtracted, for neat (a) $\mathrm{CS}_{2}$ and (b) $\mathrm{C}_{6} \mathrm{H}_{6}$.

ponent, ideally one which hardly contributes to the OHDOKE signal. ${ }^{20,27,31}$ The second motive is to understand better the dynamics of liquid mixtures themselves, in which case it is interesting to choose components that present appreciable

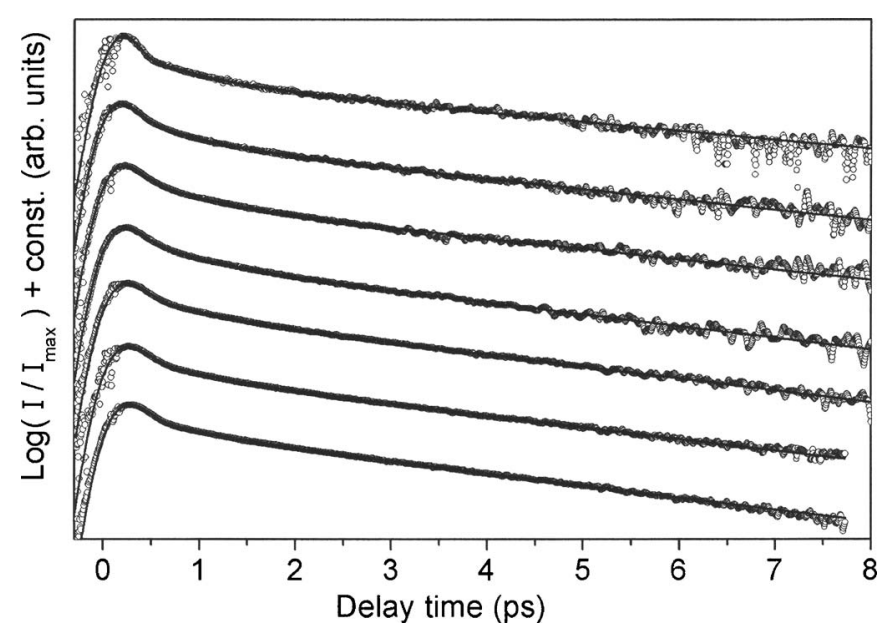

FIG. 4. Measured signal OHD-OKE (circles) for neat $\mathrm{CS}_{2}$ and $\mathrm{C}_{6} \mathrm{H}_{6}$ and for different molar fraction mixtures, together with the traces obtained by adjusting the model response function (line). From top to bottom, molar fraction of $\mathrm{C}_{6} \mathrm{H}_{6}: 1.0,0.8,0.59,0.49,0.39,0.2$, and 0.0 . The curves are offset vertically for clarity.
TABLE I. Results from the data analysis concerning the molecular dynamics of the $\mathrm{CS}_{2}-\mathrm{C}_{6} \mathrm{H}_{6}$ mixtures for the diffusive relaxation time $\tau_{d}$. Also, the measured viscosities at a temperature of $293 \mathrm{~K}$.

\begin{tabular}{ccc}
\hline \hline Molar fraction of $\mathrm{C}_{6} \mathrm{H}_{6}$ & $\tau_{d}(\mathrm{ps})$ & $\eta(\mathrm{cP})$ \\
\hline 1.00 & $2.24 \pm 0.11$ & $0.854 \pm 0.037$ \\
0.80 & $2.05 \pm 0.09$ & $0.692 \pm 0.026$ \\
0.59 & $1.99 \pm 0.07$ & $0.586 \pm 0.031$ \\
0.49 & $1.87 \pm 0.05$ & $0.530 \pm 0.047$ \\
0.39 & $1.82 \pm 0.03$ & $0.462 \pm 0.002$ \\
0.20 & $1.77 \pm 0.33$ & $0.387 \pm 0.003$ \\
0.00 & $1.75 \pm 0.02$ & $0.348 \pm 0.007$ \\
\hline \hline
\end{tabular}

interactions, and that is the trend we want to follow. Figure 4 shows the OHD-OKE transient signals for a series of decreasing benzene molar fraction mixtures in carbon disulfide. In Table I the values of the diffusive time constants obtained with the data fitting procedure is shown. These results demonstrate a gradual transition from the shorter diffusive time constant of neat $\mathrm{CS}_{2}$ (molar fraction of 0.0) until the molar fraction of 0.6 , when the diffusive time constant strongly dislocates to the longer diffusive time constant of neat $\mathrm{C}_{6} \mathrm{H}_{6}$. This modification can be explained in terms of the viscosity variation of the liquid mixtures, as can be seen in Fig. 5, which shows that the diffusive relaxation constant is a linear function of the viscosity of the mixtures (third column of Table I). The viscosity for the different molar fraction mixtures was measured at a temperature of $293 \mathrm{~K}$ with an Ostwald viscosimeter.

To analyze the nondiffusive component, we will suppose that we are primordially observing the modifications of the carbon disulfide molecular dynamics. This conjecture is based on the fact that the largest contribution to the measured OHD-OKE signal comes from the carbon disulfide molecules. To corroborate quantitatively this fact, we have realized measurements under the same experimental conditions for the two neat liquids. Figure 6 presents the spectra associated with the molecular dynamics temporal response for the different mixtures and for the pure liquids, evidencing the difference in amplitude of the carbon disulfide contribution compared to the benzene contribution [Fig. 6(a)]. The analy-

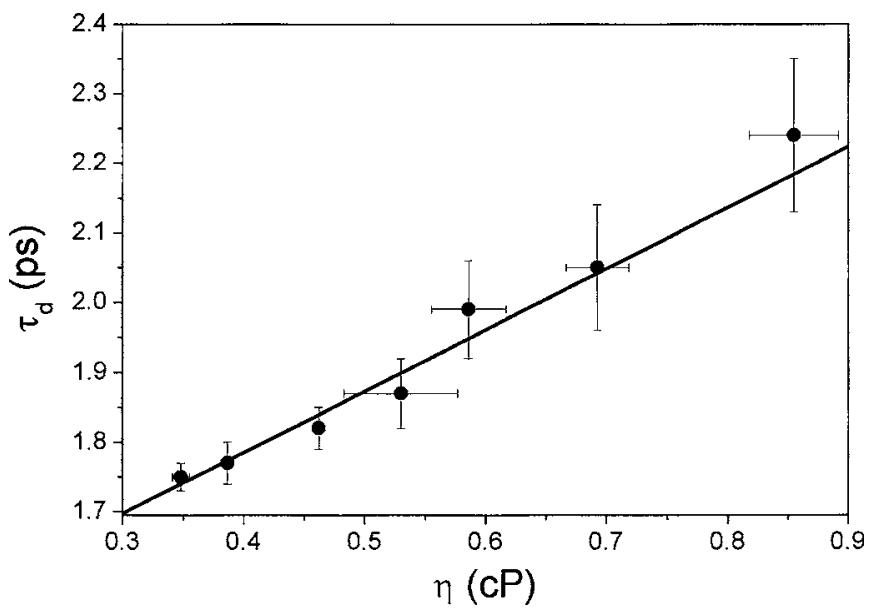

FIG. 5. Debye-Stokes-Einstein plot of the orientational diffusive correlation time $\tau_{d}$, vs viscosity $\eta$. The solid line is a linear least-squares fit to the data. 

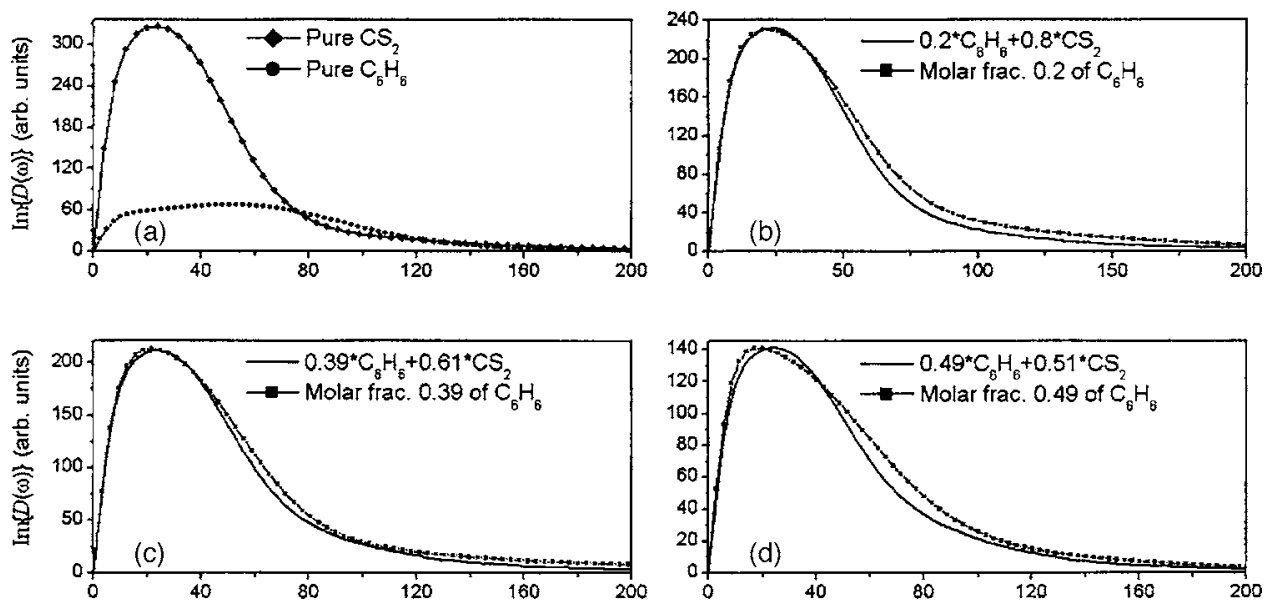

FIG. 6. The plot (a) shows the spectra associated to the molecular dynamics of the pure liquids. The other plots show the spectra obtained for the measurements of different molar fraction mixtures together with the ideal weighted averaged spectra.
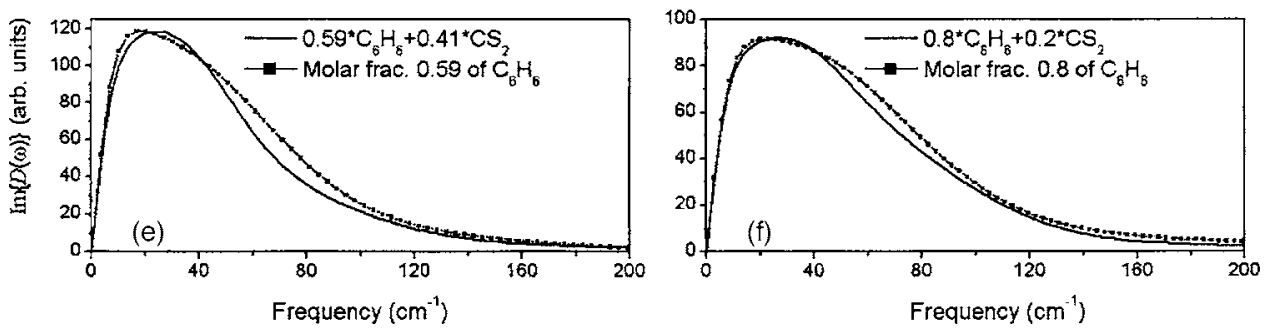

sis will be made comparatively between the ideal mixture spectrum, in which case we suppose independent contributions to the signal, and the spectrum obtained from the experimental measure. The ideal spectrum was obtained through the weighted average of the spectra measured for the pure liquids [Fig. 6(a)], using as weighting factors the molar fractions of the components of the mixture. As can be seen in Fig. 6(b), even for the $\mathrm{C}_{6} \mathrm{H}_{6}$ molar fraction equal to 0.2 , for which there is a proportion of one molecule of $\mathrm{C}_{6} \mathrm{H}_{6}$ for a group of five molecules of $\mathrm{CS}_{2}$, the spectral distribution is altered in relation to the ideal spectral distribution. To quantify the interaction induced spectral modifications we calculated the first moment of the spectral distribution, both for the measured data and for the weighted data. We also measured the full width at half maximum for the ideal and measured spectra. The results are listed in Table II, in which $\langle\omega\rangle$ and $\langle\omega\rangle_{\text {wa }}$ correspond to the first moment of the spectral distribution for the measured data of a given molar fraction and for the weighted average spectral distribution, respectively. This nomenclature is equivalent for the parameter that measures the full width at half maximum $\left(\Delta, \Delta_{\text {wa }}\right)$ of the spectral

TABLE II. First moment $\langle\omega\rangle$ and full width at half maximum $\Delta$ for the spectral distributions of the different molar fraction mixtures.

\begin{tabular}{ccccc}
\hline \hline $\begin{array}{c}\text { Molar fraction } \\
\text { of } \mathrm{C}_{6} \mathrm{H}_{6}\end{array}$ & $\langle\omega\rangle\left(\mathrm{cm}^{-1}\right)$ & $\langle\omega\rangle_{\text {wa }}\left(\mathrm{cm}^{-1}\right)$ & $\Delta\left(\mathrm{cm}^{-1}\right)$ & $\langle\omega\rangle_{\text {wa }}\left(\mathrm{cm}^{-1}\right)$ \\
\hline 1.00 & 65.08 & $\ldots$ & 95.85 & $\ldots$ \\
0.80 & 56.85 & 53.97 & 78.16 & 71.77 \\
0.59 & 51.29 & 48.69 & 67.36 & 58.10 \\
0.49 & 50.34 & 46.93 & 63.09 & 55.68 \\
0.33 & 49.30 & 45.47 & 57.10 & 53.83 \\
0.20 & 48.78 & 43.28 & 55.68 & 51.69 \\
0.00 & 43.36 & $\cdots$ & 50.57 & $\cdots$ \\
\hline \hline
\end{tabular}

distribution. The results indicate that the frequency $\langle\omega\rangle$ is always shifted to higher values in relation to $\langle\omega\rangle_{\text {wa }}$. The trend followed by the parameter $\Delta$ is a widening, as is clearly evidenced in Figs. 7(a) and 7(b).

Interpreting these results, we argue that the observed shift and widening are connected to configuration effects of the molecules inside the mixtures. When the molecules interact through different configurations, it is possible that a modification of the intermolecular potential and/or an alteration of the environmental anisotropy will occur. In a recent paper, Dani and Stassen ${ }^{32}$ presented computer simulation results based on molecular dynamics for the equilibrium properties in $\mathrm{CS}_{2}-\mathrm{C}_{6} \mathrm{H}_{6}$ liquid mixtures. Specifically they calculated the angular distribution functions that measure the orientational correlations in terms of the angles between the principal axes of symmetry of the molecules and furnish a detailed description of the local structure of the liquids. The results of that work showed firstly a perfect dilution of both compounds, since no single component clustering was noticed, which supports the fact that all solutions are optically homogeneous as observed experimentally. Secondly, it was observed, in the first solvation shell containing an average of 12 neighboring molecules, that the preferential configuration of the pairs $\mathrm{CS}_{2}-\mathrm{C}_{6} \mathrm{H}_{6}$ is perpendicular. This means that the symmetry axis $C_{\infty}$ of the $\mathrm{CS}_{2}$ molecule, which passes through the center of the carbon and sulfur atoms, stays preferentially in a direction perpendicular to the plane formed by the six carbon atoms of the benzene molecule. The distance between the centers of mass of the $\mathrm{CS}_{2}-\mathrm{C}_{6} \mathrm{H}_{6}$ pairs in this configuration was around $5.1 \AA{ }^{32}$ However, calculating the radial and angular correlations between $\mathrm{CS}_{2}$ and $\mathrm{C}_{6} \mathrm{H}_{6}$, the authors of that work found that first contact pairs orient preferentially in parallel configurations. For this configuration, 

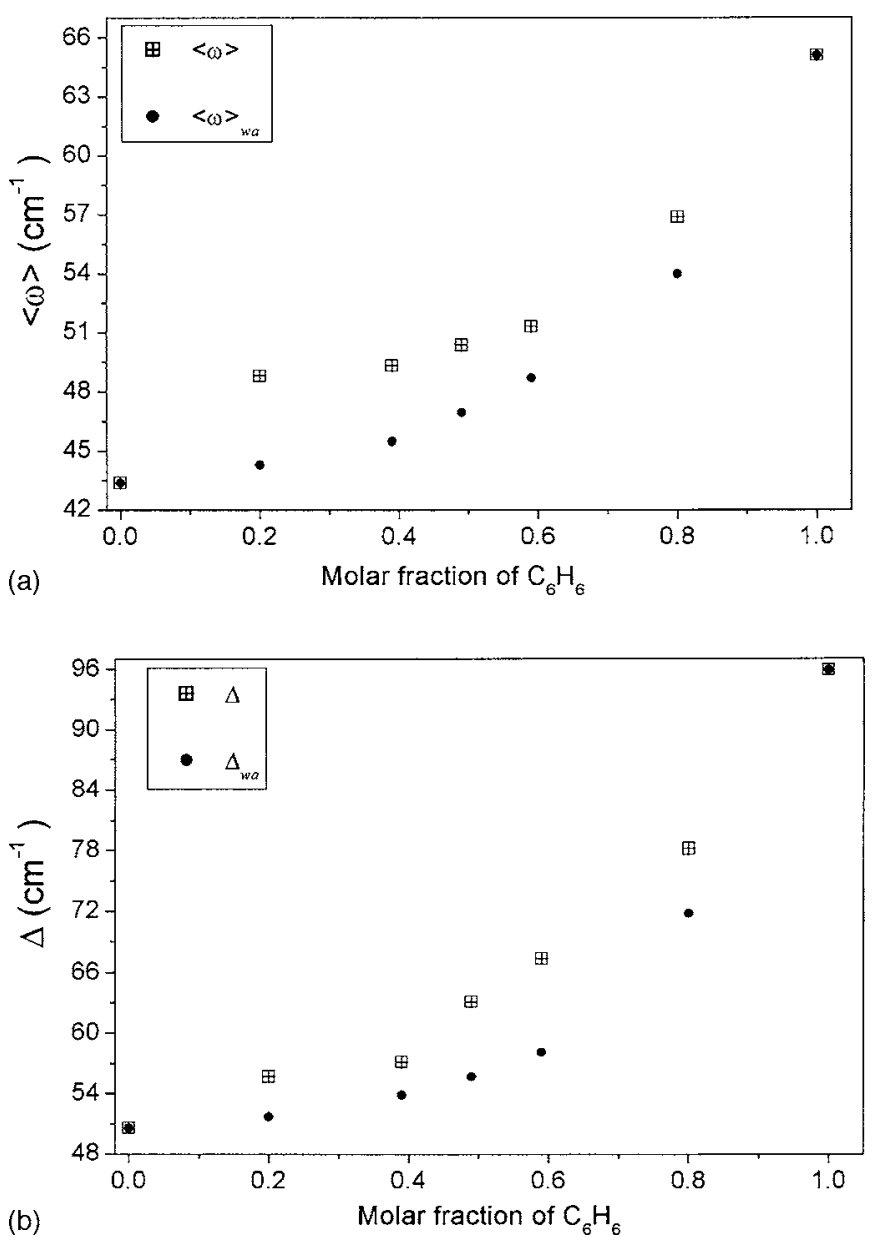

FIG. 7. Parameters associated to the spectral distributions: (a) first moment $\langle\omega\rangle$ and (b) full with at half maximum $\Delta$ for the measured signal and for the ideal weighted averaged spectra.

the distance between the centers of mass obtained was around $3.2 \AA{ }^{32}$ These results were also obtained by da Silveira et al., ${ }^{33,34}$ in which they realized an ab initio calculation on $\mathrm{C}_{6} \mathrm{H}_{6} \cdots \mathrm{CS}_{2}$ cluster to determine the stabilization energies and the preferential configurations between the $\mathrm{CS}_{2}-\mathrm{C}_{6} \mathrm{H}_{6}$ pair in this van der Waals complex. In that work, the authors also calculated the electronic density of the molecules $\mathrm{CS}_{2}$ and $\mathrm{C}_{6} \mathrm{H}_{6}$ when they were in the energy minimization configuration, establishing charge transfers between these molecules for all stable configurations. The resulting electrostatic interaction between the partially positive charged carbon atom in $\mathrm{CS}_{2}$ and the benzene $\pi$ framework contributes to the geometry stabilization. For the parallel configuration a formation of a dipole with a moment of 0.18 D occurs. $^{33}$

These theoretical results on the stabilized geometries support our proposition concerning the modification of the spectra, associated with the molecular dynamics temporal response. A new spectral feature that appears in the different molar fraction mixtures is the result of the parallel configuration of first contact pairs $\mathrm{CS}_{2}-\mathrm{C}_{6} \mathrm{H}_{6}$. Remembering our initial hypothesis that we are analyzing the modification of the $\mathrm{CS}_{2}$ molecular dynamics when these molecules interact with ever-growing molar fractions of $\mathrm{C}_{6} \mathrm{H}_{6}$ mixtures, what happens is a modification of the molecular environment that circumvent the $\mathrm{CS}_{2}$ molecules when more and more $\mathrm{C}_{6} \mathrm{H}_{6}$ molecules were added to the mixture. One needs to consider firstly that $\mathrm{C}_{6} \mathrm{H}_{6}$ molecules have a three times greater quadrupolar moment ${ }^{32}$ in relation to the $\mathrm{CS}_{2}$ molecules. In addition, they show a tendency to form dipolar structures that produce more intense intermolecular interactions. In virtue of this, the intermolecular potential inside which the $\mathrm{CS}_{2}$ molecules execute the librational motion, determined by the surrounding molecules, tends to shift to an anisotropic potential due to the reposition of the $\mathrm{CS}_{2}$ molecules by $\mathrm{C}_{6} \mathrm{H}_{6}$ molecules. This answers why the largest frequency shift for $\langle\omega\rangle$ occurs for the $\mathrm{C}_{6} \mathrm{H}_{6}$ molar fraction equal to 0.2 (an average of 2.4 benzene molecules within the first solvation shell), since this is the most anisotropic intermolecular potential case formed by preferentially parallel aligned heteromolecular pairs. The parameter that measures the full width at half maximum of the distribution $\Delta$ is related the nonhomogeneity of the intermolecular potentials in the liquid. This width has a tendency to be the broadest at the equimolar molar fractions. In this particular proportion, it is expected that there are a larger number of configuration possibilities between the molecules, since $\mathrm{CS}_{2}-\mathrm{CS}_{2}, \mathrm{C}_{6} \mathrm{H}_{6}-\mathrm{C}_{6} \mathrm{H}_{6}$, and $\mathrm{CS}_{2}-\mathrm{C}_{6} \mathrm{H}_{6}$ pairs, with most distinct stabilization geometries, would occur with equal probability for this molecular fraction.

\section{CONCLUSIONS}

Using the heterodyne detected time resolved optical Kerr effect technique, we investigated the time evolution of the molecular dynamics for a series of different molar fraction $\mathrm{C}_{6} \mathrm{H}_{6}-\mathrm{CS}_{2}$ mixtures. This technique, combined with the Fourier transform data analysis, is particularly well suited for probing low frequency intermolecular motions in liquids. The analysis of the measured data revealed that the low frequency spectra appeared broadened in comparison with the ideal mixture case. Besides, the principal librational frequency appeared shifted to higher values again in comparison with the ideal mixture case. Supported by results of molecular dynamics simulation and ab initio calculations, we concluded that these results were the consequence of structural and configuration effects that lead to an anisotropic intermolecular potential in which the $\mathrm{CS}_{2}$ molecules execute the librational motion.

\section{ACKNOWLEDGMENTS}

The authors thank the partial support of the Brazilian program PADCT III and the CNPq agency. We gratefully acknowledge Dr. Nádya P. da Silveira for helpful discussions and her student Ernesto D. Pinto for supplying us measurements concerning the mixtures viscosities.

${ }^{1}$ D. McMorrow, W. T. Lotshaw, and G. A. Kenney-Wallace, IEEE J. Quantum Electron. 24, 443 (1988).

${ }^{2}$ M. Ricci, P. Bartolini, R. Chelli, G. Cardini, S. Califano, and R. Righini, Phys. Chem. Chem. Phys. 3, 2795 (2001).

${ }^{3}$ B. J. Loughnane, A. Scodinu, and J. T. Fourkas, J. Phys. Chem. B 110, 5708 (2006).

${ }^{4}$ A. Scodinu and J. T. Fourkas, J. Phys. Chem. B 107, 44 (2003).

${ }^{5}$ D. McMorrow, N. Thantu, V. Kleinman, J. S. Melinger, and W. T. Lotshaw, J. Phys. Chem. A 105, 7960 (2001). 
${ }^{6}$ T. Steffen, J. T. Fourkas, and K. Duppen, J. Chem. Phys. 105, 7364 (1996).

${ }^{7}$ Y. Tanimura and S. Mukamel, J. Chem. Phys. 99, 9496 (1993).

${ }^{8}$ S. Mukamel, Principles of Nonlinear Optical Spectroscopy (Oxford University Press, New York, 1995).

${ }^{9}$ A. V. Pisliakov, T. Mancal, and G. R. Fleming, J. Chem. Phys. 124, 234505 (2006).

${ }^{10}$ T. Mancal, A. V. Pisliakov, and G. R. Fleming, J. Chem. Phys. 124, 234504 (2006).

${ }^{11}$ T. Steffen and K. Duppen, J. Chem. Phys. 106, 3854 (1997).

${ }^{12}$ J. C. Kirkwood, A. C. Albrecht, and D. J. Ulness, J. Chem. Phys. 111, 253 (1999).

${ }^{13}$ D. A. Blank, L. J. Kaufman, and G. R. Fleming, J. Chem. Phys. 111, 3105 (1999).

${ }^{14}$ I. A. Heisler, R. R. B. Correia, T. Buckup, S. L. S. Cunha, and N. P. da Silveira, J. Chem. Phys. 123, 054509 (2005).

${ }^{15}$ J. S. Friedman and C. Y. She, J. Chem. Phys. 99, 4960 (1993).

${ }^{16}$ M. Perrot, M. H. Brooker, and J. Lascombe, J. Chem. Phys. 74, 2787 (1981).

${ }^{17}$ F. Strehle and T. Dorfmüller, Mol. Phys. 72, 993 (1991).

${ }^{18}$ H. Stassen, PhD Thesis, Universität Bielefeld, 1992.

${ }^{19}$ R. Vilhjalmsson, Ber. Bunsenges. Phys. Chem. 85, 1071 (1981).

${ }^{20}$ C. Kalpouzos, D. McMorrow, W. T. Lotshaw, and G. A. KenneyWallace, Chem. Phys. Lett. 150, 138 (1988).
${ }^{21}$ T. Steffen, N. A. C. M. Meinders, and K. Duppen, J. Phys. Chem. A 102, 4213 (1998).

${ }^{22}$ A. Idrissi, M. Ricci, P. Bartolini, and R. Righini, J. Chem. Phys. 111, 4148 (1999).

${ }^{23}$ I. A. Heisler, R. R. B. Correia, and S. L. S. Cunha, Appl. Opt. 44, 3377 (2005).

${ }^{24}$ G. Giraud, C. M. Gordon, I. R. Dunkin, and K. Wynne, J. Chem. Phys. 119, 464 (2003).

${ }^{25}$ B. J. Berne and R. Pecora, Dynamic Light Scattering (Dover, New York, 1976).

${ }^{26}$ P. Cong, H. P. Deuel, and J. D. Simon, Chem. Phys. Lett. 240, 72 (1995).

${ }^{27}$ D. McMorrow, N. Thantu, J. S. Melinger, and W. T. Lotshaw, J. Phys. Chem. 100, 10389 (1996).

${ }^{28}$ W. T. Lotshaw, D. McMorrow, N. Thantu, J. S. Melinger, and R. Kitchenham, J. Raman Spectrosc. 26, 571 (1995).

${ }^{29}$ D. McMorrow, Opt. Commun. 86, 236 (1991).

${ }^{30}$ D. McMorrow and W. T. Lotshaw, Chem. Phys. Lett. 174, 85 (1990).

${ }^{31}$ A. Scodinu and J. T. Fourkas, J. Phys. Chem. B 107, 44 (2001).

${ }^{32}$ S. Dani and H. Stassen, J. Phys. Chem. B 107, 14000 (2003).

${ }^{33}$ N. P. da Silveira, F. S. Rodembusch, F. V. Pereira, D. Samios, and P. R. Livotto, Chem. Phys. 53, 165 (2000).

${ }^{34}$ N. P. da Silveira, H. Stassen, and T. Dorfmüller, J. Chem. Phys. 101, 9370 (1994). 